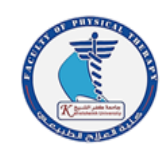

\title{
Can Functional Electrical Stimulation affect Equilibrium and motor functions in Spastic hemiparetic Cerebral Palsy? A Randomized Controlled Study
}

\author{
Abdel Aziz A. Sherief ${ }^{1 *}$, Tamer E. Elnegamy ${ }^{2}$, Mohamed K Seyam ${ }^{3}$, Mohamed A.Abdel \\ Ghafar $^{4}$ \\ ${ }^{1}$ Department of Physical Therapy for Disturbances of Growth and Developmental Disorders in \\ Children and its Surgery Faculty of Physical Therapy, Kafr El sheikh University, Egypt. \\ ${ }^{2}$ Department of Physical Therapy and health rehabilitation, College of applied sciences, Prince Sattam \\ bin Abdelaziz University, Saudi Arabia. \\ ${ }^{3}$ Department of Physical therapy and health rehabilitation, College of Applied Medical Sciences, \\ Majmaah University, AlMajmaah, Saudi Arabia. \\ ${ }^{4}$ Physical Therapy Program, Batterjee Medical College, Jeddah, Saudi Arabia.
}

\author{
*Correspondence to \\ Abdel Aziz A. Sherief \\ Ph.D of Physical Therapy, \\ Faculty of Physical \\ Therapy, Kafr El Sheikh \\ University, Egypt. \\ Tel: 01223795368 \\ Email;
}

aabdelazez10@yahoo.com

Published online:

Dec 2020

\section{Introduction}

Cerebral palsy disease is one of the most common causes to infanthood mental state deterioration and handicapping. Documentation of incidence of $\mathrm{CP}$ in developing countries is about $0.2 \%$ to $0.3 \%$ of population according to World Health Organization (WHO) (1). What usually CP patients suffering from

\begin{abstract}
:
Background and aim: Using of Functional electrical stimulation (FES) in rehabilitation program results is auspicious, although there're doubts about FES efficiency in balance and main motor functions amelioration in hemiplegic children. The research studied the impact of FES in balance and main motor skills improvement in children diagnosed with hemiparetic cerebral palsy if we placed it on ventral muscle group of leg .

Design and Place: Randomized controlled study in National Institute for NeuroMotor System in Egypt.

Methodology: 30 children who are diagnosed with hemiplegic $\mathrm{CP}$ aging from 6 to 8 years is chosen from both genders. In two equal groups randomly (control and study). Prior and after 3 months of treatment assessment was carried out, utilizing Biodex balance system to gauge static balance plus Peabody progressive motor scale to evaluate gross motor functions. Control group is put in an elected Physiotherapy program. Study group received FES as well as the elected Physiotherapy program.

Results: Study group patients showed a considerable amelioration in main motor skills test. Furthermore, in same group it's showed that balance control was better according to the other set (control group) after training .

Conclusion: Electrical stimulation addition to traditional Physiotherapy program over leg ventral group muscles can ameliorate balance and the gross motor functions in hemiparetic cerebral palsy children .

Key words: Hemiparesis; Cerebral palsy; Functional electrical stimulation and Balance.
\end{abstract}

is musculoskeletal deterioration state and wasted muscles that occur due to unknown non-progressive injury in the brain. Agonist and antagonist groups' harmony is lost, and voluntary movement control is also disrupted due to hypertonicity, so Deformations and contractures take place in $\mathrm{CP}$ patients as result to diplegic form in $\mathrm{CP}(2)$. 
Hemiplegia is the second popular form. It is a result from cerebrovascular lesion occur in utero-perinatal or postnatal stages in generality of cases. CP causes much deformation and deterioration to many physiological systems, so $\mathrm{CP}$ patients experience massive balance troubles specifically at the injured side (3). Patients also suffer from losing control in balance activities especially while standing because of hemiplegia (4). Most of children suffering CP can wake but with many restrictions (5).

It is hard for them to gain physical skills especially the progressive gross group (6). They - young patients- also can walk without assistant devices but they will walk with abnormal gait pattern (7). These anomalies comprise losing of force and significant raise in joints stiffness in push-off in extremities, the injured and non-injured legs are not symmetrical, the number of steps increase in same distance in comparison to children having other progression (8). In clinical practice Functional electrical stimulation had been utilized worldwide and get a huge regard in investigations (9). To those -incapable to contract voluntarily- muscles, FES neuromuscular applications is used within a task-specific functional activity (10).

Lately, there are more proofs provide utilization of FES due to its positive influence (11). On hemiplegic CP's patients, former study described that tibialis anterior E.S application during muscle activity leading to a significant improvement in motor efficiency and gait correction throughout just weeks later (12).

FES effectiveness and results measurement have been estimated and focused at most on structure of the body and function grade, which include, tone of muscles, power capacity, ROM of each joint and biomechanics of gait (13). Despite studies that reported results of FES utilization in rehab program are promising, there're former studies reported limited results of FES in balance control and gait asymmetry in hemiplegic children. So, this research aims to prove that electrical stimulation in addition to traditional physiotherapy program applied on leg ventral group muscles can improve balance and the gross motor functions in hemiparetic cerebral palsy children.

\section{Patients and Methods}

\subsection{Participants}

According to inclusion several criteria from clinic of Physiotherapy clinic at National Institute for neuromotor system in Egypt, thirty children of hemiparetic $\mathrm{CP}$ from both genders have been brought and they participated in the study, these criteria are: Age is from 6 to 8 years old, ability to walk freely with 5 degrees passive ankle dorsiflexion at least, capability to cope with procedures, moderate spasticity on the modified Ashworth Scale 2 or 1+ (14). And it has been excluded all children with any orthopedic surgeries for the ipsilateral limb 12 months prior to the study, Uncontrolled seizures, and botulinum poison in inferior limb 4 months before the study. Signature of the infant's parents or guardian is required on the ethical and approval form prior to the start of the study, it is important to explain the aim and measures of the study to them. The study was approved by the Ethics and research Review Committee.

\subsubsection{Randomization:}

Into two balanced sections, all participants have been distributed randomly (control and study groups) that happens after accomplishing the eligibility criteria, passing the initial assessment. Prior to being allocated randomly to either set, all children were stratified by gender. Utilizing of randomization table, randomization numbers created. To guarantee secrecy, a set of numbered, sealed, opaque envelopes will be used. Each envelope contains a card indicating which category the child is assigned to.

\subsection{Procedures of the study:}

\subsubsection{Evaluation Procedures:}

\subsubsection{Biodex balance system}

Biodex Medical System Inc, Shirley, New York, was utilized to gauge the balance from several direction. That tool includes two specific parts, the first is the mobile circle stage that allows instability for several degrees and the other is interfaced computer software monitoring. Using (APSI) anteroposterior stability index, the capability to be balanced from back to front direction is adjusted and Through (MLSI) medio-lateral stability index, the capability for balance to be controlled from side to side are both adjusted. Biodex balance system allows for eight stability levels, which ranges from stability level one (the least stable level) to stability level eight (the most stable level ). The chosen stability level is selected on the screen. In high values on the scale that means some hardness while low values is well on scale index of balance grading. To find out the capacity of the children for take control of the stage angle during tilting, the test is done. Prior to the evaluation test is applied, preparative test was given to the children to be mindful about test procedures. With lower extremities as in the stance phase of gait, the infant was ordered to stand on locked stage center. Protection support rails and biofeedback monitor changes for each infant achieved the participant's safety and comfort with the computer. Every infant could look on the screen as the presentation shows the measured setting. The child was asked to follow three paths and the mean values were determined for each pre-treatment and posttreatment participant (15). 


\subsubsection{Peabody developmental motor scale (PDMS- 2):}

This scale is utilized for simple and complicated motor functions measurement, it is extremely specific scale that are not yet completely progressed and the plane to improve fine and gross motor skills in the instructional program. This test instrument scale is standardized and has a high validity and reliability score (16).

\subsubsection{Treatment procedures:}

\subsubsection{Treatment group:}

Children in this group put on a chosen Physiotherapy program (program received Neuro developmental treatment (NDT) techniques, flexibility exercises and strength training focusing on the trunk and lower limb muscles, postural control exercise in different positions and different surfaces and general endurance training). This program applied for 12 weeks, 3 sessions per week with session time for one hour.

\subsubsection{Study group:}

Infants in this group are as same as the control group but for only 30 minutes and adding another thirty minutes applying FES over tibialis anterior for 12 weeks. During these sessions, the infants were seated on a comfortable chair, with $90^{\circ}$ knees flexion. They were barefoot, with their heels placed in contact with floor. For infants whose heels did not reach the ground, a small support box was placed under their feet. To enhance ankle dorsiflexion, the active electrode was attached to the origin of the tibialis anterior muscle. The reference electrode was attached at the insertion point of the tibialis anterior muscle. The FES device was programmed for bipolar placement at a pulse rate of 35 to $45 \mathrm{~Hz}$, on/off ratio 8 sec stimulation, $11 \mathrm{sec}$ rest. The pulse amplitude was $250 \mathrm{Mv}$. The current intensity applied was chosen during the sessions, according to the patient's sensitivity.

Evaluation for all study members before and after the study utilizing both Peabody developmental motor scale for main motor function acquisition assessment and Biodex system for balance assessment.

\section{Statistical analysis}

Data analyses were done using the SPSS software version 20. $t$ test was used to compare within and between outcome measures and also between demographic data. Chi square was utilized for comparison between genders. The significance level was set at $\mathrm{P}<0.05$. Numerical data were expressed as mean $\pm \mathrm{SD}$ and nominal data as numbers and percentage.

\section{Results}

\subsection{Demographic data:}

It was not found any considerable change in demographic data by comparison between two sets. $\mathrm{P}$ value $>0.05$ Table 1

Table (1): demographic data

\begin{tabular}{ccccc}
\multicolumn{2}{c}{ Variable } & $\begin{array}{c}\text { Control G } \\
(\mathrm{n} .=15)\end{array}$ & $\begin{array}{c}\text { Study G } \\
(\mathrm{n} .=15)\end{array}$ & $\begin{array}{c}\mathrm{P} \\
\text { value }\end{array}$ \\
\hline \multirow{2}{*}{ Age } & $\mathrm{M} \pm \mathrm{SD}$ & $7.2 \pm 0.68$ & $7.4 \pm 0.61$ & 0.996 \\
\multirow{2}{*}{ Gender } & Males & $8 / 53.3 \%$ & $9 / 60 \%$ & 0.713 \\
\cline { 2 - 5 } & Females & $7 / 46.7 \%$ & $6 / 40 \%$ & \\
\hline
\end{tabular}

M: mean, SD: stander deviation, n: number.

The results of pre-treatment demonstrated that among three parameters there is no considerable difference. (antero-posterior balance, medio-lateral balance, and locomotive subtest values of Peabody developmental motor scale) as $\mathrm{p}$ value $>0.05$.

\subsection{Antero-Posterior Balance:}

Before and after utilizing ventral dorsal balance, it is found considerable change in results value in both sets. We found that there was a statistically considerable difference between groups regarding the relation between the post mean values of the control group and the study group, promoting the study group. $\mathrm{P}$ value $<0.05$. [Figure 1]

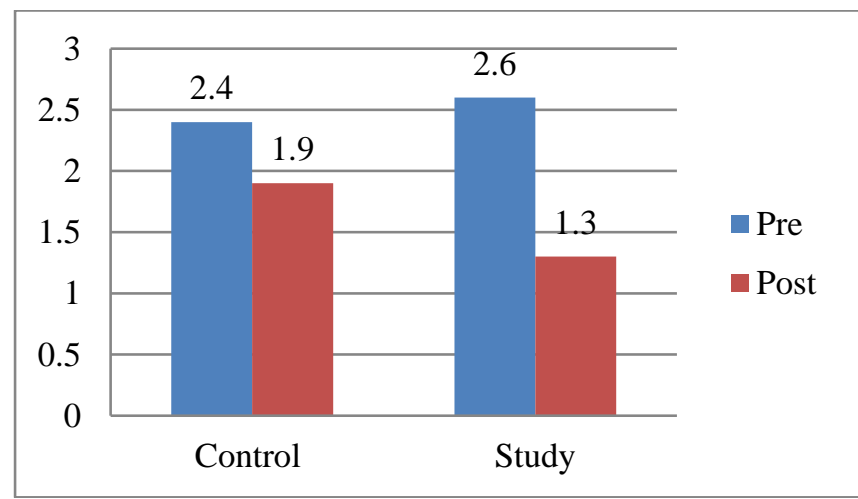

Fig.1: Comparing pre- and post-mean values of anterior-posterior balance for both groups.

\subsection{Mediolateral balance:}

In both the study sets and the control sets, there was a substantial difference between pre- and post-Medio lateral equilibrium values. Adding that there was a substantial difference between sets in the Medio lateral equilibrium measure post-value with prevailing for the study set, $\mathrm{p}$ value $<0.05$. [Figure 2] 


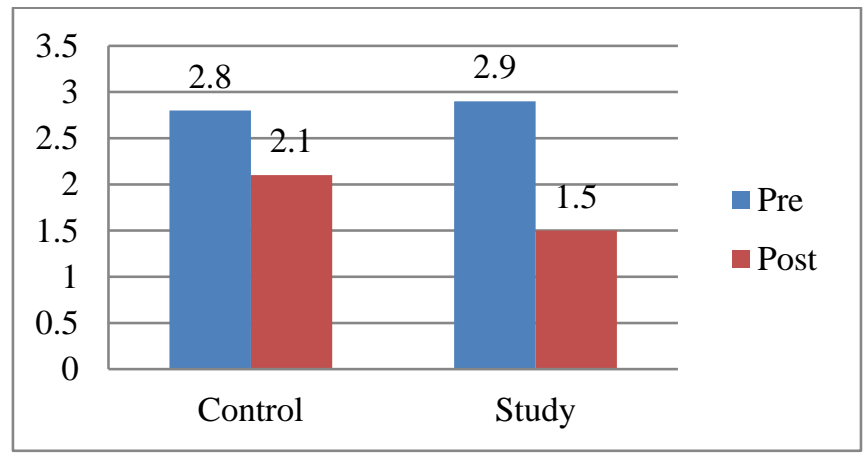

Fig.2: Comparing pre and post mean values of mediolateral balance for both groups.

\subsection{Locomotive subtest of Peabody developmental motor scale:}

Between the two sets of research the study one and the other control one, and according to Peabody progressive motor scale results it is found a considerable difference between pre- and postlocomotive subtest values. Also, with Peabody progressive motor scale concerning, there are statistically major difference between sets after measure values of locomotive subset . P value < 0.05. [Figure 3]

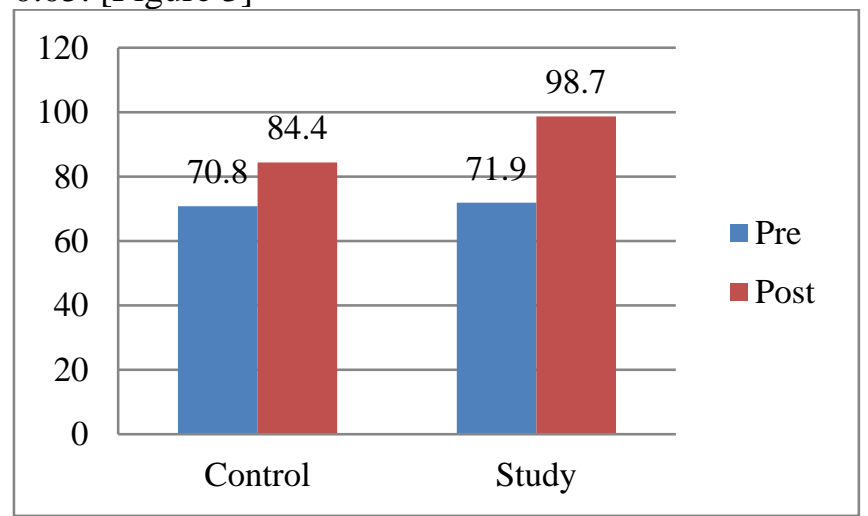

Fig.3: Comparing pre and post mean values of Locomotive subtest of PDMS-2 for both groups.

\section{Discussion}

Hemiplegic CP children is obviously having poor balance performance and gait limits due to significant weak muscles in certain body areas and decreased proprioception sensation, so they are expected to have numerous physical and motor skills ability delay because of spasticity.

This comes in agreement with Bryan \& Kevin (17) who discussed the sensory mechanism that maintain the postural balance including three parts as following, joint mechanoreceptors visual and vestibular systems. Thereafter, sensorimotor input is conveyed to the spine and brain which in turn give motor reaction that transmitted to several groups of muscles. The lesion level in hemiplegic children who are already having impaired balance is either at sensory and/or motor. As a result of CNS lesion, COG is displaced from its Base of support accurate range and it is hardly to be sensed because of spasticity.

When comparing between mean values of the two sets before and after the treatment, balance control and gait functions proved considerable amelioration at the end of the treatment program. As well as Exercising therapy for balance and postural control, Amelioration attained in the two sets may be imputed to increase muscles strength. And lower limbs coordination. This agrees with what Karimi et al. (18) finds. He discussed that to recover normal COG range in support base, Sufficient motor response needed a normal intact neuromuscular system and adequate strong muscles even in case of poor balance. Furthermore, he deduced that if system of control aimed to keep balance, it selects the appropriate motor plan of reciprocal activation. By the proprioceptive sensation and motor control system. Study show a considerable difference ( $P$ value $<0.05)$ post-treated on the two sets but more amelioration in the set of children on FES addition. These findings may be linked to the effectiveness of FES in enhancing the strength of the musculoskeletal system.

As electrical stimulation of muscle strength activates, muscle regulation of the joint and neural network is strengthened. In addition, the recovery of the mechanism of postural control and dynamic balance during weight transfer between the two limbs. Ulrich et al. (19) and Dal et al (20) sided that opinion. They mentioned that intervention of electrical stimulation especially while treadmill training will strengthen the muscle bulk, balance control and stimulate neural bundles that are critical to walk independently and balanced. Wright \& Granat (21) also agreed. They mentioned that in children with $\mathrm{CP}$ who already had FES treatment, muscles may be strengthened, and ROM maybe increase and gross motor functions will improve. Results may have different translation that necessary to be thought and it's the efficacy of FES on improve ankle dorsiflexion strength (22). It increases the active dorsiflexion angle range, affect ing kinematics gait parameter, enhancing motor control and body balance(23).

Despite FES therapeutic influence in ameliorating individual muscle or group if muscles function, Through Strength and ROM It is also studied about the whole-body function. CP patients or those who had brain lesion will suffer from absence of CNS regulation. It is resulted an abnormal pattern of gait called crouch (equine pattern) in which the knee is hyperextended, lumbar spine lordotic curve is increased and the hip is internally rotated. (24) Yang et al (25) sided that study's outcome. He reported that FES can ameliorate muscles strength and increase control of spasticity and this in turn assist ankle control and gait performance by placing 
Neuromuscular electrical stimulation on ankle dorsiflexors while walking. In study else performed by Robertson et al (26) on stroke patient. He mentioned that application of FES on ankle dorsiflexors while gait swing phase can improve balance and gait. In the other hand, this study stated utilization of FES in addition to strengthening anterior tibialis were observed. Satisfying improvement will be achieved in spastic muscle strength in hemiparetic patients even if with poor sessions, they suggested. Kerr et al (9) had review of literature and conducted. He makes analysis of how good the electrical stimulation in addition to results acquired and observed considerable improvement in tibialis anterior muscle strength. In consistent research, in which 32 children on program with FES application every day on the muscles for 2 months (27). It is shown that FES might be used to assist spastic cerebral palsy children in ambulation in easy way. This disagree with outcome of what Linden et al (28) finds. He uses application of FES on eleven $\mathrm{CP}$ children. In program containing an hour every day, for 6 days per week, for 2 months. They didn't find any considerable difference clinically or statistically in main motor skills in comparison between both groups (the control and the study one). Patient children in this study showed agonist and antagonist amelioration in both legs' muscles while cycle of gait (29).

Further research continues to explore the FES 'neural plasticity effect. The mechanisms of neural plasticity have been explored in many hypotheses, and children have greater recovery than others (29). We did not evaluate the level of neuronal plasticity directly, in this study but the assessment of the results of PDMS2 may indicate occurrence of neural plasticity, due to improvement of main motor skills after FES.

This study was limited to the small sample size, and therefore it is not conclusive.

\section{Conclusion}

Adding FES to traditional physical therapy program had better outcome on improving balance and functional capabilities in hemiplegic CP children. Finally we propose using of FES in Physiotherapy treatment program for $\mathrm{CP}$ patients in aim to considerable improvement in balance and gait.

\section{Conflict of Interests}

The authors declare no conflict of interest.

\section{Funding:}

This research did not receive any specific grant from any funding agency in the public, commercial or notfor-profit sector.

\section{References}

1. Tosun A, Gökben S, Serdaroğlu G, Polat M, Tekgül H. Changing views of cerebral palsy over 35 years: the experience of a center. Turk J Pediatr, 2013, 55: 8-15.

2. Shumway-Cook A, Hutchinson S, Kartin D, Price R, Woollacott M.Effect of balance training on recovery of stability in children with cerebral palsy. Dev Med Child Neurol, 2003, 45: 591-602.

3. Bartlett DJ and Palisano RJ. A multivariate model of determinants of motor changes for children with cerebral palsy. Phys. Ther 2002; 80: 598-614.

4. Barros CO, Roberto IT, Anizio NF, Edvin MG and Adriana BC. Balance control in hemiparetic stroke patients: Main tools for evaluation. Journal of Rehabilitation Research \& Development, 2008; 45(8): 1215-1226.

5. Bonan, I.V., Colle, F.M., Guichard, J.P., Vicaut, E., Eisenfisz, M., Tran Ba Huy, P., et al. Reliance on visual information after stroke. Part I: Balance on dynamic posturography. Archives of Physical Medicine and Rehabilitation 2004, 85: 268-273.

6. Gorter, J.W., Rosenbaum, P.L., Hanna, S.E., Palisano, R.J., Bartlett, D.J., Russell, D.J., et al. Limb distribution, motor impairment, and functional classification of cerebral palsy. Developmental Medicine and Child Neurology 2004; 46: 461-467.

7. Buckon, C.E., Thomas, S.S., JakobsonHuston, S., Sussman, M., \& Aiona, M. Comparison of three ankle-foot orthosis configurations for children with spastic hemiplegia. Developmental Medicine and Child Neurology 2001; 43: 371-378.

8. Fonseca, S.T., Holt, K.G., Fetters, L., \& Saltzman E. Dynamical resources used in ambulation by children with spastic hemiplegic cerebral palsy: relationship to kinematics, energetics, and asymmetries. Physical Therapy 2004; 84: 344-358.

9. Kerr C, McDowell B, McDonough S. Electrical stimulation in cerebral palsy: a review of effects on strength and motor function. Dev Med Child Neurol. 2004; 46:205-213.

10. Merrill DR. Review of electrical stimulation in cerebral palsy and recommendations for future directions. Dev Med Child Neurol. 2009; 51(Suppl 4(1):154-65. 
11. Meilahn JR. Tolerability and effectiveness of a neuroprosthesis for the treatment of footdrop in pediatric patients with hemiparetic cerebral palsy. PM R. 2013; 5:503-9.

12. Yea-Ru Yang, Pei-Ling $\mathrm{Mi}$, Shih-Fong Huang, Shiu-Ling,Chiu, Yan-Ci Liu, and Ray-Yau Wang, Effects of neuromuscular electrical stimulation on gait performance in chronic stroke with inadequate ankle control A randomized controlled trial. PLoS One. 2018; 13(12): e0208609

13. Damiano DL, Prosser LA, Curatalo LA, Alter KE. Muscle plasticity and ankle control after repetitive use of a functional electrical stimulation device for foot drop in cerebral palsy. Neurorehabil Neural Repair. 2013; 27(3):200-7.

14. Bohannon RW, Smith MB. Inter-rater reliability of a modified Ashworth scale of muscle spasticity. Phys Ther 1987; 67:206207.

15. Carmick, J.: Managing equinus in children with cerebral palsy: electrical stimulation to strengthen the triceps surae muscle. Dev Med Child Neurol.1995; 37: 965-975.

16. Folio MK, Fewell R. Peabody Developmental Motor Scales: Examininer's Manual. 2nd ed. Austin, Tex: Pro-Ed, Inc; 2000.

17. Bryan L. Riemann, and Kevin M. Guskiewicz. Effects of Mild Head Injury on Postural Stability as Measured Through Clinical Balance Testing. J Athl Train. 2000; 35(1): 19-25.

18. Karimi N, Ebrahimi I, Kahrizi S, Torkaman G. Evaluation of postural balance using the Biodex balance system in subjects with and without low back pain. Pak J Med Sci 2008; 6:45-52.

19. Ulrich DA, Ulrich BD, Angulo-Kinzler RM, et al. Treadmill training of infants with Down syndrome: evidence-based devel-opmental outcomes. Pediatrics 2001; 108:84-91.

20. Dal U, Erdogan T, Resitoglu B, et al. Determination of preferred walking speed on treadmill may lead to high oxygen cost on treadmill walking. Gait Posture 2010; 31:3669.

21. Wright PA, Granat MH. Therapeutic effects of functional electrical stimulation of the upper limb of eight children with cerebral palsy. Dev Med Child Neurol. 2002;42(11):724-7

22. Pool D, Elliott $\mathrm{C}$, Bear $\mathrm{N}$, et al. Neuromuscular electrical stimulation-assisted gait increases muscle strength and volume in children with unilateral spastic cerebral palsy. Dev Med Child Neurol 2016; 58: 492-501.

23. van der Linden ML, Hazlewood ME, Hillman SJ, Robb JE. Functional electrical stimulation to the dorsiflexors and quadriceps in children with cerebral palsy. Pediatr Phys Ther 2008; 20: $23-9$.

24. Strickler E, Greene W. A modified isokinetic strengthening 564 programs for patients with severe hemiplegic CP. Dev Med Child Neurol 2007; 8:121-8.

25. Yang Y-R, Mi P-L, Huang S-F, Chiu S-L, Liu Y-C, Wang R-Y. Effects of neuromuscular electrical stimulation on gait performance in chronic stroke with inadequate ankle controlA randomized controlled trial. PLoS ONE 2018, 13(12):e0208609.

26. Robertson JA, Eng JJ, Hung C. The effects of functional electrical stimulation on balance function and balance confidence in community dwelling individuals with stroke. Physiother Can. 2010; 62:114-119.

27. Dayna Pool, Jane Valentine, A. Marie Blackmore, Jennifer Colegate, Natasha Bear, Katherine Stannage \& Catherine Elliott. Daily functional electrical stimulation during every day walking activities improves performance and satisfaction in children with unilateral spastic cerebral palsy: a randomized controlled trial. (n.d.). Archives of Physiotherapy 2015, 5:5

28. van der Linden ML, Hazlewood ME, Aitchison AM, Hillman SJ, Robb JE. Electrical stimulation of gluteus maximus in children with cerebral palsy: effects on gait characteristics and muscle strength. Dev Med Child Neurol 2003; 45: 385-390.

29. Rushton DN. Functional electrical stimulation and rehabilitation - an hypothesis. Med Eng Phys. 2003;25(1):75-8 